\title{
Unsupervised Deep Learning for Blind Multiuser Frequency Synchronization in OFDMA Uplink
}

\author{
Ang $\mathrm{Li}^{1}$, Yi Ma ${ }^{1}$, Songyan Xue ${ }^{1}, \mathrm{Na} \mathrm{Yi}^{1}$, Rahim Tafazolli ${ }^{1}$, and Terence E. Dodgson ${ }^{2}$ \\ ${ }^{1}$ Institute for Communication Systems (ICS), University of Surrey, Guildford, England, GU2 7XH \\ ${ }^{1}$ E-mail: (ang.li, y.ma, songyan.xue, n.yi, r.tafazolli)@surrey.ac.uk \\ ${ }^{2}$ Airbus Defence and Space, Portsmouth, England, PO3 5PU \\ ${ }^{2}$ E-mail: terence.dodgson@airbus.com
}

\begin{abstract}
In this paper, a novel unsupervised deep learning approach is proposed to tackle the multiuser frequency synchronization problem inherent in orthogonal frequency-division multiple-access (OFDMA) uplink communications. The key idea lies in the use of the feed-forward deep neural network (FF-DNN) for multiuser interference (MUI) cancellation taking advantage of their strong classification capability. Basically, the proposed FF-DNN consists of two essential functional layers. One is called carrier-frequency-offsets (CFOs) classification layer that is responsible for identifying the users' CFO range, and another is called MUI-cancellation layer responsible for joint multiuser detection (MUD) and frequency synchronization. By such means, the proposed FF-DNN approach showcases remarkable MUIcancellation performances without the need of multiuser CFO estimation. In addition, we also exhibit an interesting phenomenon occurred at the CFO-classification stage, where the CFO-classification performance get improved exponentially with the increase of the number of users. This is called multiuser diversity gain in the CFO-classification stage, which is carefully studied in this paper.
\end{abstract}

\section{INTRODUCTION}

We consider a multiuser frequency synchronization problem in the context of orthogonal frequency-division multipleaccess (OFDMA) uplink with the following signal model

$$
\mathbf{y}=\sum_{\ell=0}^{L-1} \boldsymbol{\Omega}_{\ell} \mathbf{H}_{\ell} \mathbf{F}_{\ell}^{H} \mathbf{\Theta} \mathbf{x}_{\ell}+\mathbf{v}
$$

where $\mathbf{x}_{\ell}$ is a $(K) \times(1)$ symbol block transmitted by the $\ell^{\text {th }}$ user, which can be multiplied by a $(P) \times(K)$ precoding matrix $\Theta(P \geq K)^{1} ; \mathbf{y}$ is the received block which is the superposition of $L$ users' received signals as well as the white Gaussian noise $\mathbf{v} ; \mathbf{F}_{\ell}^{H}$ is formed by collecting $P$ columns of an $(M) \times(M)$ normalized inverse discrete Fourier transform (IDFT) matrix $\mathbf{F}^{H}$ with $\mathbf{F}_{\ell_{1}} \mathbf{F}_{\ell_{2}}^{H}=\mathbf{0}, \ell_{1} \neq \ell_{2} ; \mathbf{H}_{\ell}$ is an $(M) \times(M)$ circulant Toeplitz matrix with specific to the $\ell^{\text {th }}$ user which incorporates the propagation channel as well as the transmitter and receiver filters; $\boldsymbol{\Omega}_{\ell}$ is an $(M) \times(M)$ diagonal matrix defined by

$$
\boldsymbol{\Omega}_{\ell} \triangleq \operatorname{diag}\left[e^{\frac{j 2 \pi \omega_{\ell} L_{\mathrm{cp}}}{M}}, \ldots, e^{\frac{j 2 \pi \omega_{\ell}\left(L_{\mathrm{cp}}+M-1\right)}{M}}\right]^{T}
$$

\footnotetext{
${ }^{1}$ The precoding matrix $\Theta$ can be any user-defined matrix with full column rank. The signal model (1) is called SC-FDMA when $\Theta$ is a $(K) \times(K)$ normalized DFT matrix $(P=K)$.
}

where $\omega_{\ell}$ is the user specific carrier-frequency-offset (CFO) normalized by the symbol duration, and $L_{\mathrm{cp}}$ is the length of cyclic prefix (CP); the superscripts $[\cdot]^{T},[\cdot]^{H}$ stand for matrix/vector transpose and Hermitian transpose, respectively. Note that the linear model (1) describes the CP-removed version of the OFDMA signal to facilitate our discussion. Given the channel knowledge $\mathbf{H}_{\ell}, \forall \ell$, the aim is to determine $\mathbf{x}_{\ell}$ with minimum detection errors.

This problem has been extensively studied in the last two decades, with many results already reported in the literature. However, such seemingly saturated problem faces new challenges arising from emerging and future wireless networks. Specifically, the multiuser frequency synchronization problem involves two sub-problems. One is the estimation of multiuser CFOs, and the other is the multiuser detection (MUD) or multiuser interference (MUI) cancellation given the estimates of CFOs. The former has been well investigated concerning either the best way of using pilots/preambles [1]-[3] or statistical behaviors inherent in signal waveform [4]-[6]. However, the existing $\mathrm{CFO}$ estimation approaches face increasing challenges from the continuous growth of the user density; as it becomes increasingly difficult to provide sufficient pilot resources to support more and more users, while the signal's statistical behaviors also become hard to be exploited due to the increasing MUI. We argue for the need of novel multiuser-CFO estimators or equivalent approaches.

In the literature, the CFO estimation and MUI-cancellation problems are often considered together. Most of the MUIcancellation approaches assume the availability of CFOs estimates at the users' side (i.e., the transmitters), which facilitates MUI cancellation at the receiver through the use of transmitterside CFO pre-compensation (i.e., [3], [7]). Such trades off feedback overhead and point-to-point communication latency for the MUI-cancellation complexity. However, this approach is not suitable for $5 \mathrm{G}$ or beyond communication systems which are demanding to communication latency and feedback overhead (i.e., [8]). Given the receiver-side CFO knowledge, it is trivial to take the maximum-likelihood (ML) approach aiming at minimizing the following Euclidean distance

$$
\min _{\mathbf{x}_{\ell}}\left\|\mathbf{y}-\sum_{\ell=0}^{L-1} \boldsymbol{\Omega}_{\ell} \mathbf{H}_{\ell} \mathbf{F}_{\ell}^{H} \boldsymbol{\Theta} \mathbf{x}_{\ell}\right\|
$$


where $\|\cdot\|$ denotes the Euclidean norm. The complexity of the ML algorithm however grows exponentially with the factor of $(L K)$; and this is not practical. Alternatively, the CFO compensation can be conducted within each individual user domain (i.e., each user's sub-band in OFDMA) through the use of filterbank [5], [9], [10]. In order to mitigate the MUI, parallel interference cancellation (PIC) algorithms can be employed in an iterative manner [11]-[13]. However, the iterative MUIcancellation approaches are sensitive to the CFO estimation accuracy, and they could introduce extra computational latency on the physical layer. Therefore, we argue for the need of low-complexity low-latency MUI-cancellation method at the receiver side.

Major contribution of this paper lies in the proposal of a novel multiuser-CFO synchronization procedure; as depicted in Fig. 1. Unlike the conventional estimation-andthen-compensation procedure, the proposed approach employs a CFO classification-and-then-MUD (CAT-MUD) procedure. The CFO classification is responsible for identifying the CFO range of users. For instance, the whole $\mathrm{CFO}$ range can be divided into $Q$ sub-ranges denoted by $\Psi_{q}=\{\omega \mid q \Delta \omega \leq \omega<$ $(q+1) \Delta \omega\}, \quad q=0, \cdots, Q-1$, where $\Delta \omega$ defines the size of the sub-range. A CFO classifier is employed to tell which subrange(s) the users fall in. This differs from the coarse CFO estimation in the sense that the latter yields CFO estimates, but the classifier only gives a range index $q$. After the CFO classification, the received signal $\mathbf{y}$ is fed into a corresponding MUD component included in the MUI-cancellation layer for the MUI cancellation and signal recovery, and the multiuser frequency synchronization problem is naturally resolved.

The proposed CAT-MUD procedure is realized by employing a feed-forward deep neural network (FF-DNN). As depicted in Fig. 2, the FF-DNN consists of two functional layers. One is called the CFO classification layer, which plays the role of CFO classification. The other is called the MUIcancellation layer, which takes the role of joint MUD and frequency synchronization. The motivation of employing FFDNN lies in the fact that:

- Both CFO classification and MUD functions involved in the CAT-MUD procedure are typically the classification problem. This is where FF-DNN can play a central role. The use of FF-DNN for MUD can be found in the literature [14], [15]; and in this paper we will offer a further study.

- The FF-DNN after training can yield a low-complexity solution to the CFO classification and MUD. Moreover, the FF-DNN structure is naturally parallel, and thus it supports parallel computing. All of these meet the requirements for low-complexity low-latency receiver design.

We train the proposed FF-DNN with the unsupervised learning approach. It is found that the CFO-classification layer and the MUI-cancellation layer work together in the communication procedure though, they can be trained separately at the learning stage; and there is no performance penalty. In the communication procedure, it is discovered that the CFO classification performance can improve exponentially with the number of users in the CFO sub-range. This phenomenon is called the multiuser diversity gain. However, the multiuser diversity-gain does not contribute considerably to the MUD performance when the system goes beyond a certain number of users (i.e., $L \geq 4$ in mobile fading channel). This means that the CFO classification accuracy is already sufficient, and further improving the accuracy would not improve the MUD performance. Finally, the proposed CAT-MUD approach is compared with the current approaches in terms of block error rate (BLER), and the former demonstrates remarkable performance gain.

\section{Preliminaries AND PRinciples}

The use of deep learning for signal detection (i.e., MUD) is equivalent to the classification of received signals $\mathbf{y}$ according to the maximum a posteriori (MAP) principle [14]

$$
\hat{\mathbf{x}}_{\ell}=\underset{\mathbf{x} \ell}{\arg \max } p\left(\mathbf{x}_{\ell} \mid \mathbf{y}, \mathbf{H}_{\ell}\right), 0 \leq \ell \leq L-1
$$

where $p(\cdot)$ denotes the probability. Given the channel knowledge at the receiver side, the performance of signal detection (or classification) is dominated by two factors: 1) the white Gaussian noise v, and 2) the uncertainty of CFOs. The former has been extensively investigated, and the latter is the problem of interest.

To facilitate our study, we define a vector $\mathbf{z}$ as the noiseless version of $\mathbf{y}$, i.e.,

$$
\begin{aligned}
\mathbf{z} & \triangleq \sum_{\ell=0}^{L-1} \boldsymbol{\Omega}_{\ell} \mathbf{H}_{\ell} \mathbf{F}_{\ell}^{H} \boldsymbol{\Theta} \mathbf{x}_{\ell} \\
& =\sum_{\ell=0}^{L-1} \boldsymbol{\Omega}_{\ell} \mathbf{F}^{H} \boldsymbol{\Lambda}_{\ell} \mathbf{F} \mathbf{F}_{\ell}^{H} \boldsymbol{\Theta} \mathbf{x}_{\ell}
\end{aligned}
$$

where $\mathbf{H}_{\ell}=\mathbf{F}^{H} \boldsymbol{\Lambda}_{\ell} \mathbf{F}$ follows the Fourier decomposition, and $\boldsymbol{\Lambda}_{\ell}$ is the diagonal matrix with its diagonal entries to be the channel frequency responses (CFR). We further define an $(M) \times(M)$ matrix

$$
\mathbf{G} \triangleq\left[\boldsymbol{\Omega}_{0} \mathbf{F}^{H}, \ldots, \boldsymbol{\Omega}_{L-1} \mathbf{F}^{H}\right]
$$

and an $(M) \times(1)$ vector

$$
\mathbf{s} \triangleq\left[\mathbf{x}_{0}^{T} \boldsymbol{\Theta}^{T} \overline{\boldsymbol{\Lambda}}_{0}, \ldots, \mathbf{x}_{L-1}^{T} \Theta^{T} \overline{\boldsymbol{\Lambda}}_{L-1}\right]^{T}
$$

where $\overline{\boldsymbol{\Lambda}}_{\ell}$ is a sub-matrix of $\boldsymbol{\Lambda}_{\ell}$ with its diagonal entries selected by $\left(\mathbf{F F}_{\ell}^{H}\right)$. Then, (6) can be written into a compact matrix form

$$
\mathbf{z}=\mathbf{G s}
$$

Proposition 1. Given the matrices $\boldsymbol{\Theta}$ and $\boldsymbol{\Lambda}_{\ell}, 0 \leq \ell \leq L-1$, the CFO uncertainty will result in ambiguity in the signal detection (or classification) when there exists at least two different pairs $\left(\mathbf{G}_{1}, \mathbf{s}_{1}\right),\left(\mathbf{G}_{2}, \mathbf{s}_{2}\right)$ such that

$$
\mathbf{z}=\mathbf{G}_{1} \mathbf{s}_{1}=\mathbf{G}_{2} \mathbf{s}_{2}
$$

Proposition 1 indicates a case where the received signal cannot be well classified through MAP or ML principles; and 


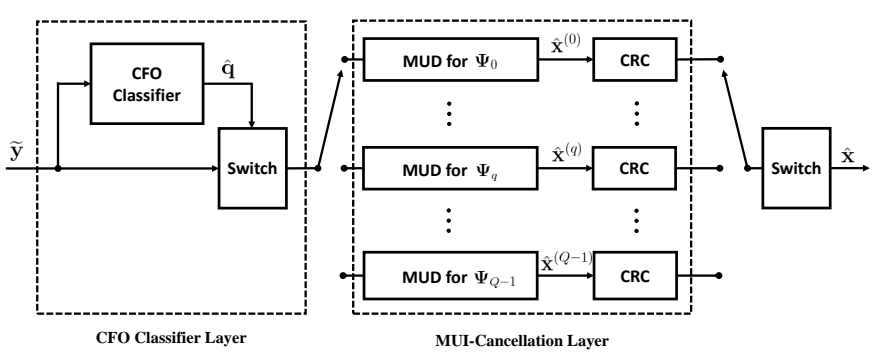

Fig. 1. CAT-MUD Block Diagram

this is also the case where deep learning can hardly handle. As shown in [15], the classification ambiguity is related to the size of CFO sub-range $\Delta \omega$. When $\Delta \omega \leq 0.05$, the probability of the case (10) is reasonably small for the signal classification. Although there is lack of rigorous proof due to mathematical intractability, the similar result has been reported in other experimental works [16], [17]. Hence, we can summarize the observation in previous works into the following statement

Proposition 2. Suppose: c1) $\Delta \omega$ is sufficiently small; and c2) the relationship (10) holds. There exists a unique combination of $\mathbf{G}$ and $\mathbf{s}$ given $\mathbf{z}$, i.e., $\mathbf{G}_{1}=\mathbf{G}_{2}, \mathbf{s}_{1}=\mathbf{s}_{2}$.

In light of this observation, we assume: A1) all active users have their CFOs fulfilling $\omega_{\ell} \in \Psi_{q}, \forall \ell$. Then, the multiuser frequency-synchronization procedure can be divided into two steps: s1) to identify the sub-range $\Psi_{q}$; and s2) to perform the MUD. The first step is called the CFO classification, which can also follow the MAP principle

$$
\hat{q}=\underset{q}{\arg \max } p\left(\Psi_{q} \mid \widetilde{\mathbf{y}}_{0}, \ldots, \widetilde{\mathbf{y}}_{N-1}\right)
$$

where $\widetilde{\mathbf{y}}_{n}, n \in[0, \ldots, N-1]$, stands for the received signal block with $\mathrm{CP}$ inserted; and $N$ denotes the number of received blocks.

Proposition 3. The probability of $\mathrm{CFO}$ misclassification is inversely proportional to $\Delta w$, and goes asymptotically to zero with the increase of $\Delta \omega$, i.e., $p(\hat{q} \neq q) \rightarrow 0$ with $|\Delta \omega| \rightarrow 0.5$.

Given that the random CFO varies continuously, for those located at the edge of the CFO sub-range i.e., $\Psi_{q}$, inevitably, there exists the non-zero probability with which the CFO classifier drops the received signal into the sub-range i.e., $\Psi_{q \pm 1}$, adjacent to the ideal CFO sub-range. Thus, the unideal MUD component is assigned to handle the MUI-cancellation. It is obvious that this CFO misclassification probability can be minimized by sufficiently enlarging the CFO sub-range $\Delta \omega$. However, the classification ambiguity in the MUD procedure becomes consequently more significant which degrades more the system performance. Therefore, $\Delta \omega$ needs to be carefully set up so as to well balance the trade-off between the CFO classification precision and MUD reliability.

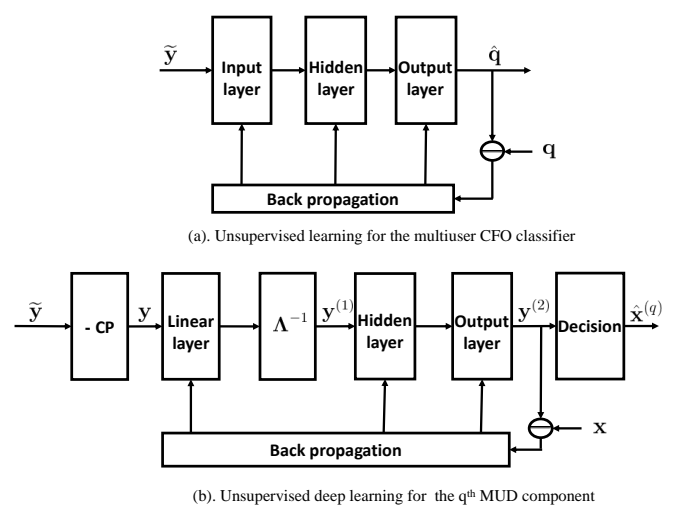

Fig. 2. CAT-MUD Neural Network Structure

\section{The CAT-MUd Procedure AND Its DeeP LEARNING REALIZATION}

\section{A. CAT-MUD Procedure}

Fig. 1 illustrates the block diagram of the CAT-MUD procedure. The proposed procedure is basically formed by two functional layers. The first functional layer includes the multiuser CFO classifier with its neural network structure depicted in the Fig. 2 (a). Specifically, it consists of three fully-connected layers. The target of the CFO classifier is to tell the sub-range $\Psi_{q}$ where $\widetilde{\mathbf{y}}$ falls in. Note that the block index $n$ in (11) is omitted here since the CFO classifier is able to give the prediction based on only one received block. Therefore, the classification procedure follows the simplified MAP principle.

$$
\hat{q}=\underset{q}{\arg \max } p\left(\Psi_{q} \mid \widetilde{\mathbf{y}}\right),
$$

The output is formed by a $(Q) \times(1)$ vector $\hat{\mathbf{q}}$ with its $q^{\text {th }}$ element indicating the highest probability of $\widetilde{\mathbf{y}}$ falling into the corresponding CFO sub-range $\Psi_{q}$. Then the CPremoved version $\mathbf{y}$ is fed into the MUD component which correspondingly takes the charge of MUI-cancellation and MUD for $\Psi_{q}$.

Fig. 2 (b) illustrates the block diagram of one of the parallel MUD components that have same structure. After CPremoval, the received signal distorted by the channel and thermal noise is successively fed into two linear layers for the MUI-cancellation and channel equalization, which can be mathematically expressed by

$$
\begin{aligned}
\mathbf{y}^{(1)} & =\boldsymbol{\Lambda}^{-1}\left(\mathbf{W}^{(1)} \mathbf{y}+\mathbf{b}\right) \\
& =\boldsymbol{\Lambda}^{-1} \boldsymbol{\Gamma} \mathbf{y}
\end{aligned}
$$

Where $\Gamma$ represents the linear transform in the first linear layer and $\boldsymbol{\Lambda}^{-1}$ is the channel inverse matrix with its diagonal submatrix entries defined by

$$
\boldsymbol{\Lambda}^{-1} \triangleq \operatorname{diag}\left[\overline{\boldsymbol{\Lambda}}_{0}^{-1}, \ldots, \overline{\boldsymbol{\Lambda}}_{L-1}^{-1}\right]^{T}
$$

As it was proved in [15], finding the appropriate linear transform $\Gamma$ helps minimizing the multiuser signal classification error. Since multiuser CFOs $\omega_{\ell}, \forall \ell$ are involved and 
lead the optimization of $\boldsymbol{\Gamma}$ to an integer linear programming problem which is NP-complete, the deep learning algorithm is employed to seek for a near-optimum solution.

The following hidden layer and the output layer are jointly responsible for MUD. Basically, the MUD procedure follows the MAP principle in (4). Note that every MUD component is independent to each other and able to detect all users' signal i.e., the $q^{\text {th }}$ MUD component detects all users' signals and outputs the recovered signal in the form of $\hat{\mathbf{x}}^{(q)}=$ $\left[\hat{\mathbf{x}}_{0}^{(q) T}, \ldots, \hat{\mathbf{x}}_{L-1}^{(q) T}\right]^{T} . \hat{\mathbf{x}}_{\ell}^{(q)}, \forall \ell$. The recovered is considered as correct if it passes the following CRC.

\section{B. Deep Learning for CAT-MUD Optimization}

1) Multiuser $C F O$ Classifier Optimization: The unsupervised learning procedure for the $\mathrm{CFO}$ classifier is illustrated in Fig. 2 (a). The weighting matrix and bias vector in each layer are updated through mini-batch gradient descent algorithm combined with Adam optimizer [18]. The activation function for the hidden layer is ReLU and the one for the output layer is Softmax with the output value of the neurons summing up to 1 . The received signal $\widetilde{\mathbf{y}}$ is converted into real-equivalent version so as to facilitate the computation [19]. A training sample $(\hat{\mathbf{q}}, \mathbf{q})$ is defined as the pair of the output value $\hat{\mathbf{q}}$ and the one-hot vector $\mathbf{q}$ with its '1-bit' index corresponding to the index of CFO sub-range where the received signal falls in. The loss is calculated through the cross-entropy function for each training sample pair and then the average of the sum over 1000 pairs' loss is used to update the weighting matrix and bias for each training iteration. The classifier is trained to handle the CFO $\omega_{\ell} \in[-0.5,0.5], \forall \ell$ where each sub-range covers $\Delta \omega=0.05$ for the sake of performance trade-off between $\mathrm{CFO}$ classification and MUI-cancellation. It is worth noting that all users CFOs fall in the same sub-range throughout the whole training set $\left(\geq 2 \times 10^{5}\right.$ iterations) so as to allow the learning curve to converge. The training SNR is set to be $7 \mathrm{~dB}$ for AWGN and $15 \mathrm{~dB}$ for fading channel as this configuration is found the best for the performance.

2) MUD Component Optimization: Fig. 2 (b). illustrates the unsupervised deep learning procedure for one of the DNNbased MUD components. Each MUD component includes four layers in which the first two layers are linear and the rest are non-linear layers. Similar to the optimization procedure for the multiuser CFO classifier, the MUD component using mini-batch gradient descent combined with Adam to iteratively optimize the weighting matrix and bias vector. Note that the channel inverse matrix $\boldsymbol{\Lambda}^{-1}$ in the first hidden layer is fixed in each training epoch and varies based on to users' channel states. The training process is only applied to the MUD component that is responsible for the CFO sub-range $\Psi_{0}=\left\{\omega_{\ell} \mid 0 \leq \omega_{\ell}<0.05\right\}, \forall \ell$ since the difference between different MUD components is just the frequency shift. The optimization for other MUD components can be simply completed by multiplying the relevant frequency shift by $\boldsymbol{\Gamma}$ in the MUD component for $\Psi_{0}$.

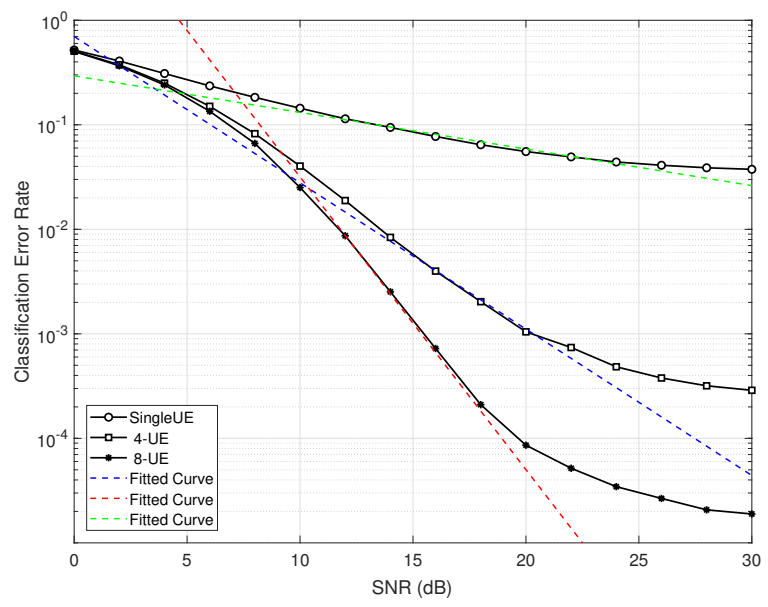

Fig. 3. Probability of CFO misclassification as a function of SNR (dB) in the AWGN channel

\section{Simulation Results and Performance EVALUATION}

The main objective of computer simulations is to evaluate the performance of the proposed CAT-MUD procedure in OFDMA uplink communications. Consider an OFDMA system in the simulation which is configured by: $M=32$ OFDM subcarriers, CP length of $8, L=1,4$ or 8 with each user being allocated by $(M) /(L)$ consecutive OFDM subcarriers. The modulation scheme are either BPSK or grey-coded 16QAM. Communication channels are either the AWGN or the 8-tap Rayleigh fading channel. Throughout the simulations, the precoding scheme is considered as an identity matrix with the same size $\Theta=\mathbf{I}$. Note that the simulation results are obtained through sufficient Monte-Carlo trials.

Experiment 1: This experiment aims to evaluate the multiuser-CFO classification performance in both AWGN and mobile fading channels, where the unknown CFOs for all users are set to fulfil $\omega_{\ell} \in \Psi_{q}, \forall q, \ell$.

Fig. 3 illustrates the probability of CFO misclassification, $P_{e}$, for BPSK modulation as a function of signal-to-noise (SNR) in AWGN, where the number of users varies. It is observed that:

- Generally, the multiuser-CFO classification accuracy improves with the increase of SNR. The performance improvement is relatively slow at lower SNRs $(<8 \mathrm{~dB})$, due to the impact of noise; or higher SNRs $(>20$ $\mathrm{dB})$, due to the the intrinsic ambiguity inherent in the CFO classification procedure; as already discussed in Proposition 3. At the typical SNR range $(8<$ SNR $<20$ $\mathrm{dB})$, the performance improves almost linearly with the SNR.

- The performance of multiuser-CFO classification improves significantly with the increase of users. To understand this interesting phenomenon, we conduct linear curve fitting at the SNR range, where the classification errors degrade linearly. It is found that that the probability 


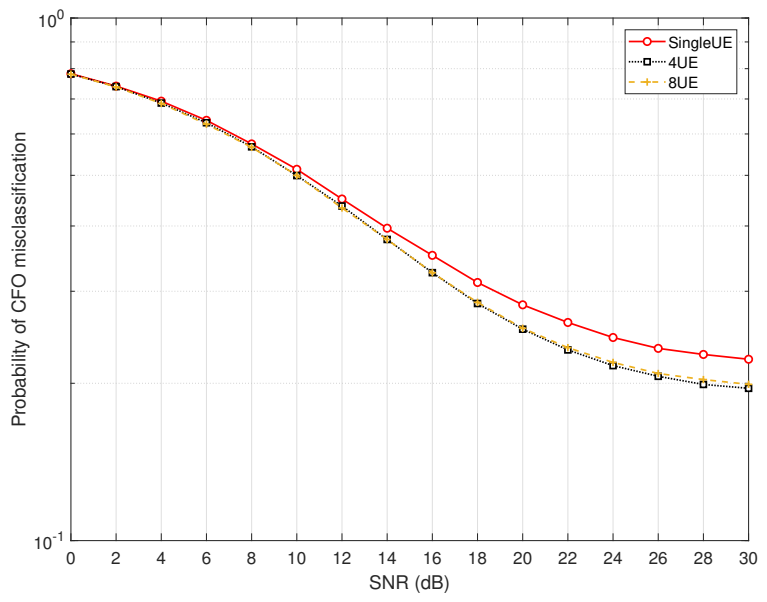

Fig. 4. Multiuser CFO classification-error-rate as a function of SNR (dB) over mobile fading channels

of misclassification can be expressed by

$$
P_{e}=\alpha_{L} \mathrm{SNR}^{-0.35 L}
$$

where $\alpha_{L}$ denotes the coefficient related to the number of users $\left(\alpha_{1}=0.29, \alpha_{4}=0.7\right.$ and $\left.\alpha_{8}=20\right)$; the SNR here is measured in the linear scale. We call such an interesting behaviour the multiuser diversity gain, where $L$ is the diversity order.

It is worthwhile to highlight that the multiuser diversity gain comes from the CFO diversity of different users. The users, with their CFOs located at the edge of the CFO range $\Psi_{q}$, have higher probability of misclassification, and those, with their CFOs at the centre of the CFO range, have much lower probability of misclassification. Our experimental results show that the DNN determines the CFO range based on a certain voting mechanism, and this is where the diversity gain comes from. However, the voting algorithm used by DNN is hard to be observed; and this could be a piece of interesting future work.

As far as fading channels are concerned, the multiuser diversity gain appears to be less significant because the fading channel effect corrupts the amplitude and phase of the received signals, and this could confuse the CFO classifier. As illustrated in Fig. 4, the multiuser diversity gain appears from 12 $\mathrm{dB}$ and saturates at $20 \mathrm{~dB}$. Different from the phenomenon shown in the AWGN channel, increasing the number of users does not show a remarkable contribution to the CFO classification accuracy in the Rayleigh fading channel, i.e., nearly identical probabilities of misclassification observed in both 4-user and 8-user scenarios throughout the SNRs.

It is perhaps worth noting that the DNN-based CFO classifier is able to identify the CFO range for OFDM signals in the presence of CPs, but not for those having their CPs removed. It means that the DNN-based classifier was actually exploiting the cyclo-stationarity induced by $\mathrm{CPs}$ for the $\mathrm{CFO}$ classification.

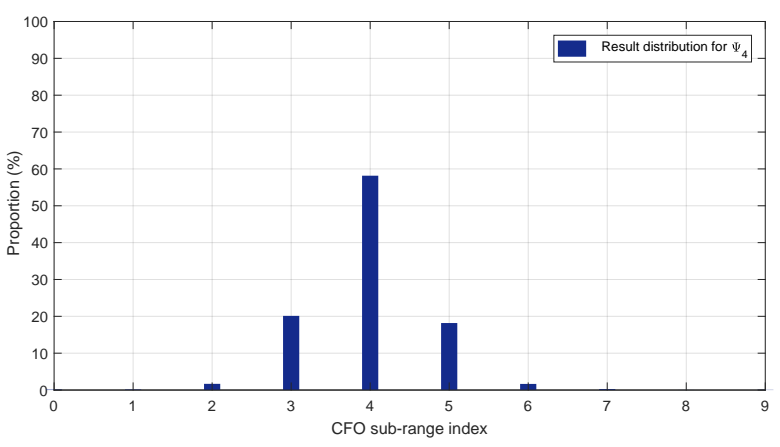

Fig. 5. Historgram Multiuser CFO classification in mobile fading channel

Experiment 2: The experiment aims to investigate the detailed result of what the classifier is able to do in the CFO classification procedure, given that the CFOs fall in a specific CFO sub-range. Note that $\mathrm{CFO}$ the classification results are gathered in sufficient Monte Carlo trials under the mobile fading channel at the fixed SNR of $15 \mathrm{~dB}$.

Due to the channel effect, the CFO classification accuracy is significantly degraded. Fig. 5 illustrates the histogram of the 4-UE CFO classification result for a specific CFO sub-range, i.e., $\Psi_{4}$. It is observed that nearly $57 \%$ of the classifications correctly fall into $\Psi_{4}$ while nearly $38 \%$ are dropt into the adjacent subr-ranges, i.e., $\Psi_{3}$ and $\Psi_{5}$. Unideal CFO classification may lead to the incorrect assignment of the corresponding MUD component to conduct the MUI cancellation and detection. In order to ensure the correct MUD component to be assigned, a parallel-MUD (PMUD) scheme based on the existing CAT-MUD structure is proposed, where numbers of MUD components is activated simultaneously. For example, based on the classified CFO sub-range $\Psi_{4}$, the corresponding MUD component for $\Psi_{4}$ and its adjacent MUD components responsible for $\Psi_{3}$ and $\Psi_{5}$ are simultaneously activated to work in parallel. By doing so, the correct MUD can be guaranteed with a probability of $95 \%$.

Experiment 3: This experiment aims to evaluate the performance of the proposed CAT-MUD procedure. The baseline used for comparison is the conventional PIC CFOcompensation method [12].

Fig. 6 illustrates the user-averaged block-error-rate (BLER) performance as a function of $E_{b} / N_{0}(\mathrm{~dB})$ in the OFDMA uplink communication over Rayleigh fading channels. It is observed that each user has nearly identical performance and only the averaged BLER results are shown. The BLER is measured for each individual users' signal block. We observed from the result:

- Three-component CAT-PMUD significantly outperforms the single-component CAT-MUD scheme, i.e., $3.5 \mathrm{~dB}$ detection gain observed at $E_{b} / N_{0}$ of $15 \mathrm{~dB}$. The performance gain is exploited by the PMUD scheme because on the one hand, it is more likely for the three-component CAT-PMUD scheme than the CAT-MUD scheme to con- 


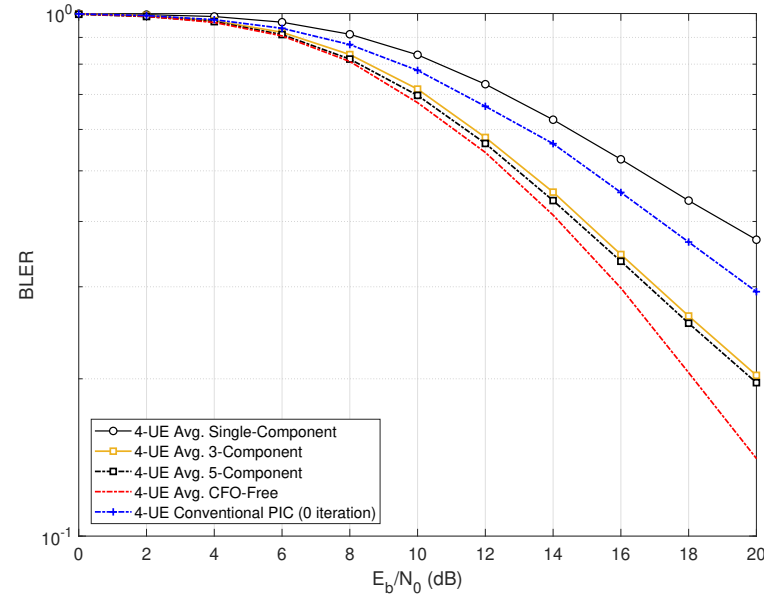

Fig. 6. BLER performance for 4-UE OFDMA uplink 16-QAM CAT-MUD as a function of $E_{b} / N_{0}(\mathrm{~dB})$ over mobile fading channels

duct the ideal MUD as discussed previously. On the other hand, the suimultaneously activated MUD components enhance the detection reliability [15]. It means that the MUD component covering $\Psi_{q}$ is able to conduct the signal detection when users' CFOs are located at the edge of $\Psi_{q}$ or even outside the sub-range.

- The small performance improvement $(\leq 0.5 \mathrm{~dB})$ is observed in the five-component scheme compared to the three-component PMUD scheme. This observation shows that the sequence detection gain comes from the most adjacent two MUD components. Therefore, the best set-up for the CAT-PMUD scheme is to activate three parallel MUD components simultaneously, which is able to offer the reliable MUD performance and avoid high computational complexity.

\section{CONCLUSION}

In this paper, a novel unsupervised deep learning approach has been presented to handle the blind multiuser frequency synchronization problem in OFDMA uplink communications. The proposed approach replaced the conventional CFO estimation and compensation procedure with a novel CFO classification and multiuser detection procedure. It has been shown that the DNN-based CFO classifier and MUD components can be trained separately, and they can operate together to offer a remarkable MUD performance. Moreover, it is found that the CFO classifier can exploit the multiuser diversity to improve the $\mathrm{CFO}$ classification performance.

\section{ACKNOWLEDGEMENT}

This work was partially supported by the Airbus project on Artificial Intelligence for Communications, and partially by the UK HEFEC-funded 5G Innovation Centre.

\section{REFERENCES}

[1] P. Sun and L. Zhang, "Low complexity pilot aided frequency synchronization for OFDMA uplink transmission," IEEE Trans. Wireless Commun., vol. 8, no. 7, pp. 3758-3769, July 2009.

[2] Y. Zeng and A. R. Leyman, "Pilot-based simplified ML and fast algorithm for frequency offset estimation in OFDMA uplink," IEEE Trans. Veh. Technol., vol. 57, no. 3, pp. 1723-1732, May 2008.

[3] M. Morelli, "Timing and frequency synchronization for the uplink of an OFDMA system," IEEE Trans. Commun., vol. 52, no. 2, pp. 296-306, Feb 2004.

[4] J. J. van de Beek, M. Sandell, and P. O. Borjesson, "ML estimation of time and frequency offset in OFDM systems," IEEE Trans. Signal Process., vol. 45, no. 7, pp. 1800-1805, Jul 1997.

[5] J. J. van de Beek et al., "A time and frequency synchronization scheme for multiuser OFDM," IEEE J. Sel. Areas Commun., vol. 17, no. 11, pp. 1900-1914, Nov 1999.

[6] S. Barbarossa, M. Pompili, and G. B. Giannakis, "Channel-independent synchronization of orthogonal frequency division multiple access systems," IEEE J. Sel. Areas Commun., vol. 20, no. 2, pp. 474-486, Feb 2002.

[7] Y. Ma and R. Tafazolli, "Estimation of carrier frequency offset for multicarrier CDMA uplink," IEEE Trans. Signal Process., vol. 55, no. 6, pp. 2617-2627, June 2007.

[8] P. Popovski, J. J. Nielsen, C. Stefanovic, E. d. Carvalho, E. Strom, K. F. Trillingsgaard, A. Bana, D. M. Kim, R. Kotaba, J. Park, and R. B. Sorensen, "Wireless access for ultra-reliable low-latency communication: Principles and building blocks," IEEE Netw., vol. 32, no. 2, pp. 16-23, March 2018.

[9] J. Choi, C. Lee, H. W. Jung, and Y. H. Lee, "Carrier frequency offset compensation for uplink of ofdm-fdma systems," IEEE Commun. Lett., vol. 4, no. 12, pp. 414-416, Dec 2000.

[10] D. Huang and K. B. Letaief, "An interference-cancellation scheme for carrier frequency offsets correction in OFDMA systems," IEEE Trans. Commun., vol. 53, no. 7, pp. 1155-1165, July 2005.

[11] M. O. Pun, M. Morelli, and C. C. J. Kuo, "Iterative detection and frequency synchronization for OFDMA uplink transmissions," IEEE Trans. Wireless Commun., vol. 6, no. 2, pp. 629-639, Feb 2007.

[12] M. Movahedian, Y. Ma, and R. Tafazolli, "Iterative carrier frequency offset estimation and compensation for OFDMA uplink," in Proc. IEEE 19th Int. Symp. on Personal, Indoor and Mobile Radio Commun. (PIMRC), Sept 2008, pp. 1-5.

[13] M. Movahhedian, Y. Ma, and R. Tafazolli, "Blind CFO estimation for linearly precoded OFDMA uplink," IEEE Trans. Signal Process., vol. 58, no. 9, pp. 4698-4710, Sept 2010.

[14] S. Xue, Y. Ma, and R. Tafazolli, "Unsupervised deep learning for MU-SIMO joint transmitter and noncoherent receiver design," IEEE Wireless Commun. Letts., 2018. [Online]. Available: http: //epubs.surrey.ac.uk/848911/

[15] A. Li, Y. Ma, S. Xue, N. Yi, and R. Tafazolli, "A carrier-frequencyoffset resilient OFDMA receiver designed through machine deep learning," in Proc. IEEE 29th Annual Int. Symp. on Personal, Indoor and Mobile Radio Commun. (PIMRC), Sept. 2018. [Online]. Available: http://epubs.surrey.ac.uk/848624/

[16] A. Felix, S. Cammerer, S. Dörner, J. Hoydis, and S. T. Brink, "OFDMautoencoder for end-to-end learning of communications systems," in 2018 IEEE 19th Int. Workshop on Signal Process. Advances in Wireless Commun. (SPAWC), June 2018, pp. 1-5.

[17] S. Dörner, S. Cammerer, J. Hoydis, and S. t. Brink, "Deep learning based communication over the air,' IEEE J. Sel. Topics Signal Process., vol. 12, no. 1, pp. 132-143, Feb 2018

[18] D. P. Kingma and J. Ba, “Adam: A method for stochastic optimization," CoRR, vol. abs/1412.6980, 2014

[19] J. C. D. L. Ducoing, N. Yi, Y. Ma, and R. Tafazolli, "Using real constellations in fully- and over-loaded large MU-MIMO systems with simple detection," IEEE Wireless Commun. Letts., vol. 5, no. 1, pp. 9295, Feb 2016. 